\title{
AUTOMATED PROPER MOTION SURVEY: DATA REDUCTION AND INITIAL SYSTEM PERFORMANCE
}

\author{
A. E. La Bonte \\ Control Data Corporation
}

\section{INTRODUCTION}

The Automated Proper Motion Survey (APMS) has three broad goalsaccuracy, completeness, and efficiency in the discovery and measurement of stellar proper motions on pairs of red-sensitive photographic star plates taken with the forty-eight inch Schmidt telescope. The specific range of motions sought is from 0.1 to 2.5 seconds-of-arc per year. The lower limit of 0.1 arc sec/year is consistent with the inherent uncertainties in the photographic emulsion and the typical epoch difference between plate exposures. At the opposite end of the scale, extension of the search radius beyond that corresponding to $2.5 \mathrm{arcsec} /$ year would result in a prohibitively large number of spurious matches and a significant increase in processing time while yielding extremely few (though individually interesting ) additional stellar motions. The specific range of stellar magnitudes sought is from 12 to 19 red. Significant motions for stars brighter than the limit $\mathrm{m}_{\text {red }}=12$ are already fairly well documented and the corresponding bright Sčfmidt images begin to show extensive contamination from diffraction spikes, "blazes" radially away from the plate center, and photographic "bloom". At the other limit, although images of stars fainter than $m$ red $=19$ are visually discernible on the plates (the plate limit is typically $\mathrm{m}_{\mathrm{red}}^{\mathrm{red}}=20$ ), inspection of the faintest images reveals that they are amorphous and often quite asymmetric clusters of photographic grain. Thus, both the motion limits and the magnitude limits for the survey have been selected to cover the range of reliable and largely unexplored data on the plate material. The implementation of APMS, then, is tailored to these goals and ranges.

The APMS system consists of (1) the set of star plate pairs; (2) a general purpose star-plate scanner which detects, encodes, and records image scan data on computer compatible magnetic tape; and (3) a data reduction software package which analyzes the scan data to discover, measure, and assess stellar motions. The system is designed to exploit both the input from the tremendous parallel data acquisition capacity of the Schmidt Telescope and the extensive computational capabilities of large-scale highspeed general purpose digital computers; this compination allows all threg APMS goals to be emphasized. The plate material $(1,2)$ and the scanner $(3)$ have been extensively described elsewhere and in the remainder of this introduction, only those facets which are needed as a frame of reference are summarized. The body of the paper then concentrates on the third major element of the system - the data processing scheme - and reports the initial performance results from the system as a whole. 


\section{A. Star Plate Characteristics}

The National Geographic Society - Mount Palomar Observatory Sky Survey covers the entire celestial sphere north of declination $-33^{\circ}$ ( or 77 percent of the sky) with $936 \mathrm{red} /$ blue plate pairs taken with the forty-eight inch Schmidt telescope. Duplicates of the red plates from this original survey constitute the set of initial epoch plates for the proper motion survey. The second epoch data consists of repeats of these red plates on the same centers but with a typical time lapse of 13 years; this repetition is now nearly complete.

The second epoch or new plates are photographic "originals" - that is, the actual plates exposed in the telescope. As a consepuence, the new plates tend to have a high and variable level of background fog and slightly granular images. The old plates, on the other hand, have gone through two photographic duplication processes which has "hardened" all but the faintest images (below the survey limit). Further, the processing was controlled so that the duplicates exhibit a much clearer and uniform background. For both new and old plates, however, the images in the magnitude range 13-19 red appear very round and preserve this property over the entire plate. This superb image quality lends itself to very efficient algorithms for reconstructing and assessing the stellar data. Images from stars in the remainder of the survey magnitude range, 12-13 red, show incipient diffraction spike structure; they do not possess detectable asymmetry, however, and are essentially round except in the vicinity of the spikes. An image diameter range of 220 microns to 40 microns on the old (duplicate) plates is found to bracket the survey magnitude range of $12-19$ red. The new plates were taken under somewhat more variable conditions so the appropriate diameter range on these plates is found through correlation with the corresponding old plates. Finally, images brighter than $\mathrm{m}_{\mathrm{red}}=12$ are of interest as references of known position for establishing the conversion from plate to celestial coordinates. As noted above, these bright images possess a variety of structural anomalies and image centers accurate enough for proper motion determinations often cannot be inferred. Nevertheless, good centers can be obtained for many individual images of stars as bright as eighth magnitude; this appears to provide more than adequate overlap with the reference data in the Smithsonian Astrophysical Observatory Star Catalog $(4)$ Indeed, there are sufficient bright image-catalog matches to overdetermine the coordinate transformation very strongly and the effects of random errors in the center determinations are reduced statistically.

The plate scale is very nearly 15 microns/arcsec. Thus, for an average epoch difference of 13 years, the motion range of $0.1-2.5 \mathrm{arcsec} /$ year converts to an image displacement range of approximately $20-500$ microns. 
The image counts on the plates range from $3 \times 10^{4}$ on the very sparsest plates near the galactic poles to values of the order of 10 for some low galactic latitude regions which are not obscured by gas clouds. Most of the plates, however, contain a few hundred thousand images and at this density, the number of overlapping images among the stars in the survey range is small. The large bright star images, of course, do blot out much of the area in their immediate vicinity but the number of these stars per plate pair is small. Thus, for the majority of the survey, the images of interest may be treated as isolated circular dots.

A much more serious problem is presented by plate "flaws" of various kinds. Particularly on the new plates, the high background fog results in random clumps of photographic grain which are not readily distinguishable from some of the smaller stellar images. Emulsion and plate imperfections are also sometives difficult to discriminate against. It has been estimated empirically that the number of small "round" flaws is comparable to the counts of legitimate images on the sparser plates. Thus, the data reduction software must contain methods for dealing with the large number of spurious matches likely to result from the presence of these imperfections in the plate material; a catalog of "moving" plate flaws is clearly not desired. Finally, long scratches perpendicular to the direction of scan often show up and give rise to many spurious data points in the stream of scan data. Although it is very improbable that this data will be mistaken for stellar image information (unless the scratch is broken into many very short segments), it is important from an efficiency standpoint that the software be capable of recognizing and discarding such data at the earliest possible point in the processing.

\section{B. Scanner Characteristics}

The star plate scanner is designed as a fairly general purpose plate inter rogator which will sample each "image" on the plate in sufficient detail to characterize the location, size, and some structural classification for that image. The image sample along with the APMS scanner coordinate system is illustrated in Figure 1; all quantities shown are encoded with a granularity of one micron.

Although the image sample is conceptually simple, the scanner implementation must gather this data accurately and at high speed. The electronics must be capable of handling a very broad range of average data rates and an even broader range of instantaneous or burst rates. In addition to accomodating the random arrival rate of the stream of transit detections, the acquistion logic must compress the data sample with efficient encoding and transfer uniform compact blocks of data to magnetic tape in a computer compatible format. Note that such time smoothing of the data is extremely important for efficient data reduction; large fixed blocks of compressed 


\section{APMS SCANNER COORDINATE SYSTEM}

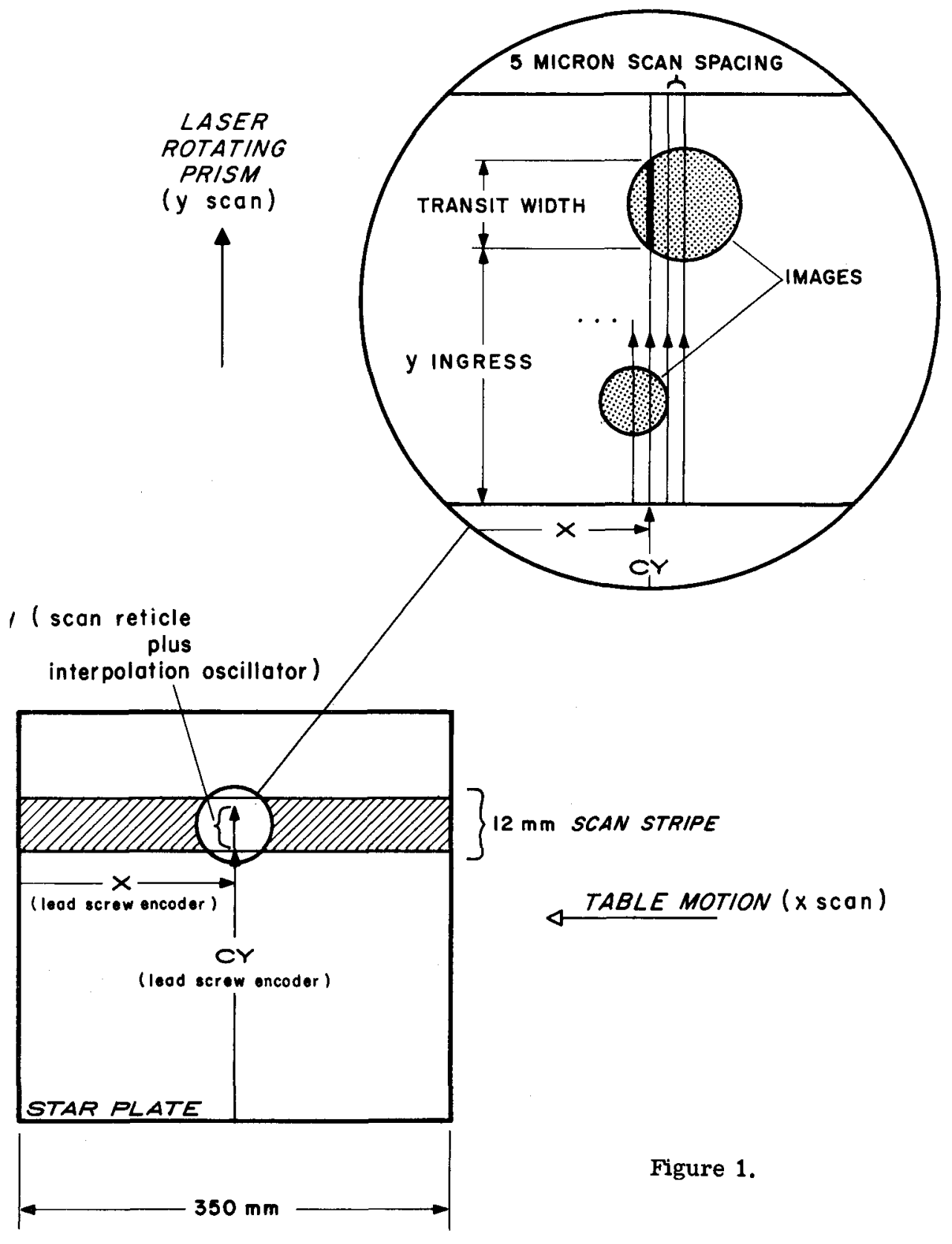


information insure rapid data transfer and permit the system software to operate within a deterministic input enviroment.

The manner in which the image samples are manipulated and the type of information which is inferred from them will vary for different types of problems; such variations would be implemented as modifications to the software. This approach, with a relatively general but fixed scanner and flexible software, provides the potential of adapting the system to new problems at relatively low expenditure. Perhaps more importantly, however, it allows the present system to evolve as the problem and the plate material become better understood through experience. With this brief background then, the discussion turns to the current implementation of the APMS software.

\section{APMS PROCESSING STRUCTURE}

The principal APMS processing steps as outlined in Figure 2 are divided into a pre-production calibration and verification phase and a production plate processing cycle. This latter cycle is applied to each plate pair in the survey and begins with the plate scan operation. Next, a small portion of the encoded information is analyzed to check the quality of the data and to establish values for several plate-dependent control parameters. This "pilot" operation insures correct and efficient performance of the full plate reduction which follows as the third step of the cycle. Here, the complete set of scan data is reduced on a "local" basis with only a small portion of one stripe (typically a $12 \mathrm{~mm} \times 12 \mathrm{~mm}$ segment) considered at any instant. The output from this process is a list of apparent motions plus a statistical compilation of image characteristics from the "fixed" stars on the plate and a list of the bright images which were successfully reconstructed from the scan data.

The bright image list and the fixed star compilation each cover a statistically large sample which is used in the fourth step of the cycle to characterize the "global" properties of the plate data. The bright images are matched with corresponding SAO catalog stars to infer the plate-tocelestial coordinate transformation. The fixed star statistics describe typical stellar image characteristics for each plate individually and establish the plate-to-plate correlation between matched pairs of stellar images; all of these statistics are determined as a function of image size. The resultant global data is then used to transform and assess each individual motion candidate. This process classifies the apparent motions into those which may be accepted a priori, those which may be immediately discarded because they exhibit one or more serious deviations from the expected properties, and a small set which lie on the borderline. 


\section{PRINCIPAL APMS PROCESSING STEPS}

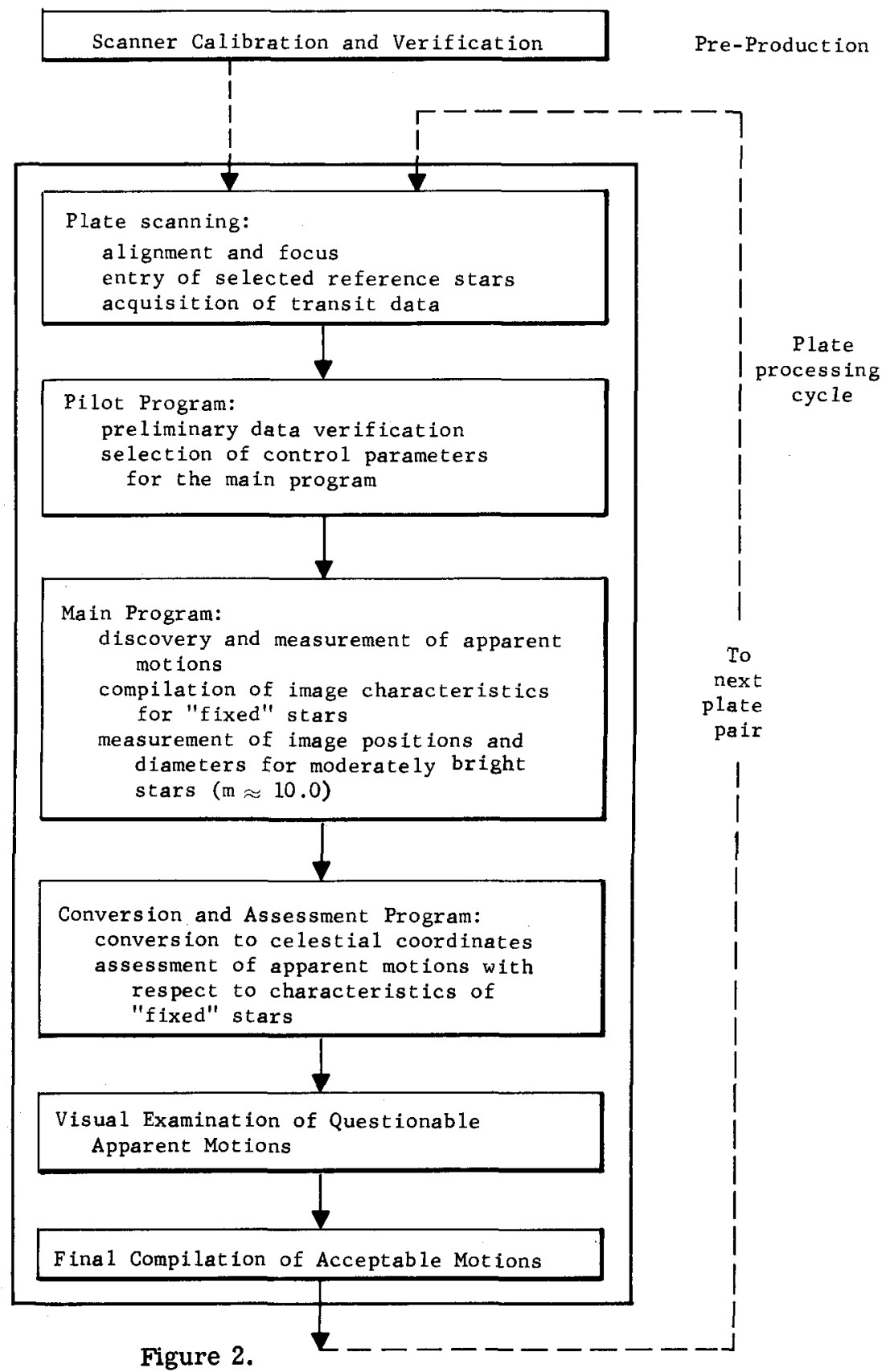


At the fifth step in the cycle, the questionable motion candidates are referred back to the astronomer for visual resolution. Finally, all acceptable motions for the plate pair are tabulated and published; the process then recycles to the next plate pair.

This completes the overview of the APMS processing structure. From the discussion above, it is clear that the total APMS software package must enter at several different steps in the flow and must carry out rather diverse operations. Further, it is often desirable or even necessary to interpret the results between steps and to take action on the basis of the interpretation. Thus, the APMS software system is implemented as a series of computer programs including three calibration programs, a program to extract the reference stars for a particular plate pair from the SAO catalog tape, the pilot program, the main "local" discovery and measurement program, the "global" assessment and conversion program, and a short program to compile the final results.

The heart of the process and by far the major computer time requirements are embodied in PROG RAM APMSM, the main discovery and measurement program. The flow through A PMSM ( Figure 3 ) begins with the operation of reading and unpacking a block of compressed data from the scanner generated magnetic tape. Each block contains information from only one of the two simultaneously scanned plates in the pair while data within the block is ordered sequentially along the scan path. Thus, the next operation consists of sorting the transits from scan order into groups, each corresponding to a separate "image".

The image groups are then subjected to the first of several filters designed to eliminate spurious or contaminated data at the earliest possible point in the processing. The transit count filter accepts only those groups for which the total number of transits falls between prescribed upper and lower bounds. In order to maximize data compression efficiency, the scanner rejects any transit longer than approximately one millimeter. Thus, the largest legitimate symmetric image which can be accepted should contain fewer than 200 transits and the upper count limit is set at this value. This limit is very effective in deleting data from scratches. On the other hand, images at the faint limit of the survey are still large enough to contain several transits - at least six on the old duplicate plates and typically five or six on the new photographic original plates. The limit for the new plate is determined by the nature of its correlation with the old duplicate; this limit is one of the control parameters established by the pilot program results. In any event, groups containing very few transits are discarded completely and those containing just fewer than the limit are processed in a special manner. The lower limit of the transit count filter throws out a great deal of spurious data associated with isolated detections of the photographic background grain or other small flaws. 


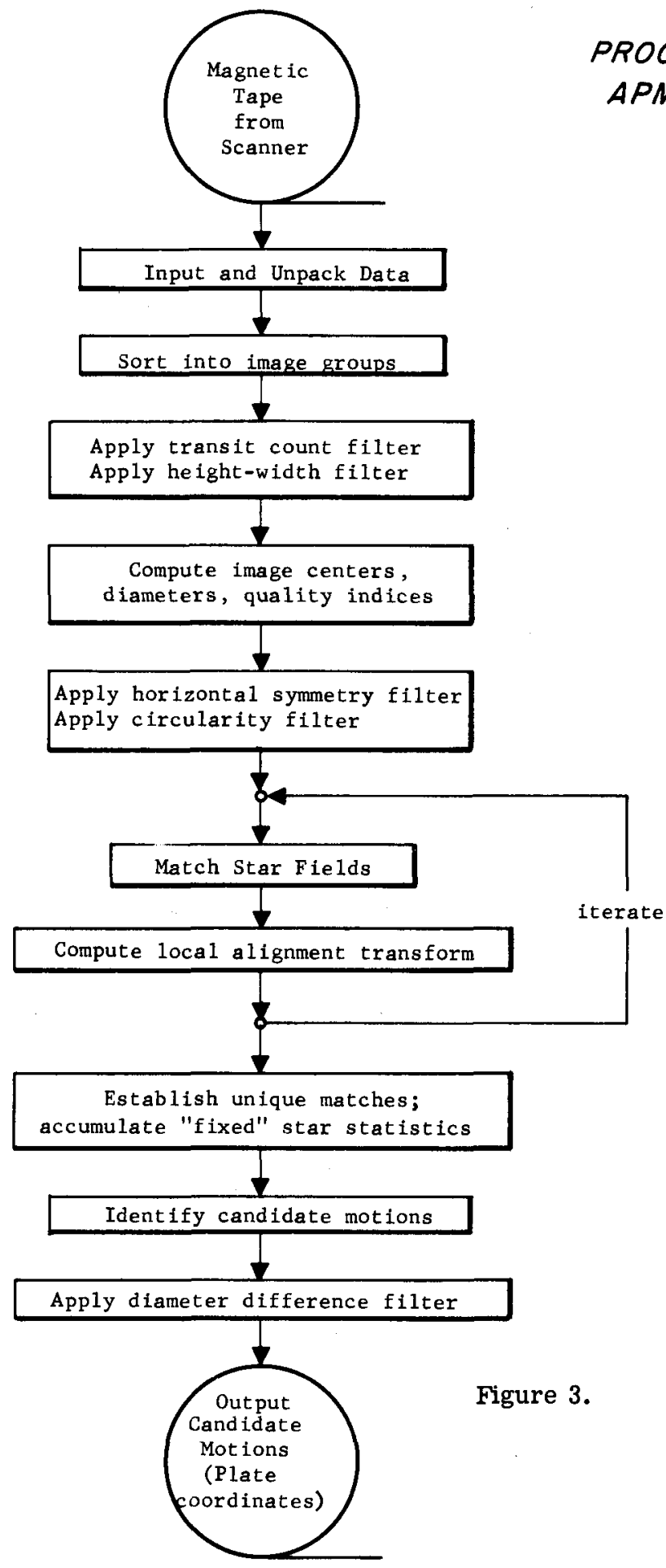


The special processing for groups just below the lower count limit takes into account the fact that the plate material exhibits considerable magnitude variation and that a sharp uniform cutoff is not possible. Thus, the cutoff for the old duplicate may discard an image which is retained on the new plate and vice versa. This would leave several legitimate but unmatched images which might then enter in to spurious apparent motion matches. Therefore, a crude and computationally inexpensive center is estimated for each borderline group and these centers are stored in a marginal image array for further checking in the match process.

As noted earlier, the images of interest for the survey are very cir cular; the height and width of the image should be very nearly equal. The image height may be approximated by the longest transit in the group and the image width by the number of transits in the group times the scan spacing. These two measures are very inexpensive to obtain and the height-width filter forms an effective means for deleting short scratch segments and other images which are significantly non-circular (or more properly non"square").

The image groups which pass through these first two filters come from images which are at least potentially circular. In the next major processing step, $\mathrm{x}$ and $\mathrm{y}$ positions of the image center, the image diameter, and two image quality indices are inferred for each accepted group. The algorithm for estimating the y center is particularly simple; the midpoint of each individual transit is itself an estimate for the y center and the average of these midpoints over the entire group provides an excellent direct value. Further, if the image is truly circular, then it will certainly be symmetric about a horizontal line through its center. The average absolute y deviation of the individual transit midpoints from the y center estimate for the group is therefore a measure of the horizontal symmetry of the image and is taken as the first quality index.

Inference of the $x$ center and the size of the image is not as straightforward. One possibility is to solve for $\mathrm{x}$ center and the radius jointly (given the y center ) by fitting the transit end points to a circle in a least-squares sense. The solution to this problem can be written down in closed form. However, the resulting expression is computationally expensive given the number of images to which it must be applied. In an alternate approach, a set of equations is again written which assumes that each transit endpoint lies on a common circle of unknown radius and $x$ center; but in this case the equations are differenced in pairs. Endpoints from different, wellspaced transits are used in each pair and the differencing process deletes both the radius-squared and the $x$ center-squared terms from the resultant relation. This leaves an equation in which $\mathrm{x}$ center enters only linearly and is the only unknown; a simple direct estimate for $\mathrm{x}$ center results. The $\mathrm{x}$ 
center estimate actually used, however, is obtained from the average over a set of such pair-difference equations involving all transit endpoints in the group. The algorithm produces values only tenths of microns less accurate than the least squares solution and is very efficient.

Once the $\mathrm{x}$ and $\mathrm{y}$ center positions are known, the square of the image radius is estimated by averaging the image center-to-transit endpoint values over the entire group. Again, if the image is truly circular and an accurate center has been found, the distance from the center to the various endpoints should be invariant. The average absolute radius deviation of the individual transit endpoints is therefore a measure of the circularity of the image and is taken as the other quality index.

Filters are then applied to both quality indices and images which are highly asymmetric or acircular are transferred to the marginal image array; they may, of course, correspond to legitimate but contaminated stellar images.

The above processing continues until all raw scan data within the current stripe segment has been reduced. For each plate, this yields a list of the approximately circular images with known quality indices, diameters, and image centers which lie within the segment. A list of the marginal images is also available. This completes the intraplate reduction for the stripe segment.

At the next stage, the lists of accepted images from the two plates are searched to establish several tentative image pairings. This process is aided significantly by careful visual alignment of the plates in the scanner prior to the original scan data acquisition. Such alignment insures that image mates of non-moving stars will appear at nearly coincident points in the streams of scan data from the two plates. Under these circumstances a fairly small initial search radius can be used and few ambiguities will arise.

The match step is followed by determination of a mathematical transformation to refine the plate alignment. The transform computation is based on the list of tentative image pairs established above but will initially be distorted by inclusion of motion star pairs and very occasional image mismatches in the list. Thus, the match and alignment steps are iterated with an increasingly tighter match tolerance as the transformation improves. The iterations are continued until only "fixed" stars are used in establishing the final transformation.

It may be noted here that a general affine transformation is employed; it will account for translational and rotational misalignments plus stretch 
and skew between the fields. This functional form appears to be adequate since non-linear effects from emulsion stretch and warp appear to be negligible over a typical $12 \mathrm{~mm} \times 12 \mathrm{~mm}$ stripe segment. Such effects are operative over larger distances on the plates, however, as evidenced by the fact that complete visual alignment cannot be established simultaneously over the entire plate.

The final transformation is applied to every image in the field and all "unique" matches are established between the lists of acceptible images on the two plates. Here, a unique match is one in which the relative displacement is less than the lower motion limit (typically 20 microns). Such matches are almost certainly correct pairings of stellar images. As a consequence, the corresponding image quality indices and the plate -to-plate diameter correlation among these pairs are accumulated to provide the "fixed" star statistics. The remaining list of unmatched accepted images on each plate is then checked against the marginal image list for the other plate in an effort to delete other unique matches. Here, a slightly larger match tolerance is used since the centers of the marginal images are less reliable. Also, characteristics from these matches are not included in the fixed star statistics.

Next, the two resultant lists of acceptable but still unmatchedimages are searched for all matches with separations between the minimum and maximum motion limits. The pairs which arise here are submitted to one last filter which rejects matches with very large diameter differences between the component images. Pairs passing this filter then represent the motion candidates from the current segment and all associated data is finally written on the output tape. The entire process, of course, is repeated until all segments of the plate have been reduced in the search for and measurement of stellar proper motions.

\section{APMS INITIAL PERFORMANCE}

A general approach to the APMS problem has been outlined and several aspects of the APMS software have been presented. The subject of prime interest, however, is the performance of the system in the survey task; the remainder of the paper describes the initial results obtained in the program.

Following the scanner calibration, a series of system verification tests were undertaken. The first of these was designed to evaluate the magnitude sensitivity of the scanner. The old/new plate pair P 322 which covers SA 57 was employed for the investigation; this Selected Area contains the only well established magnitude sequence which extends down to the plate limit. Images of stars with known photoelectric magnitudes and colors $(5,6)$ were individually identified on the plate and the photoelectric data was used 


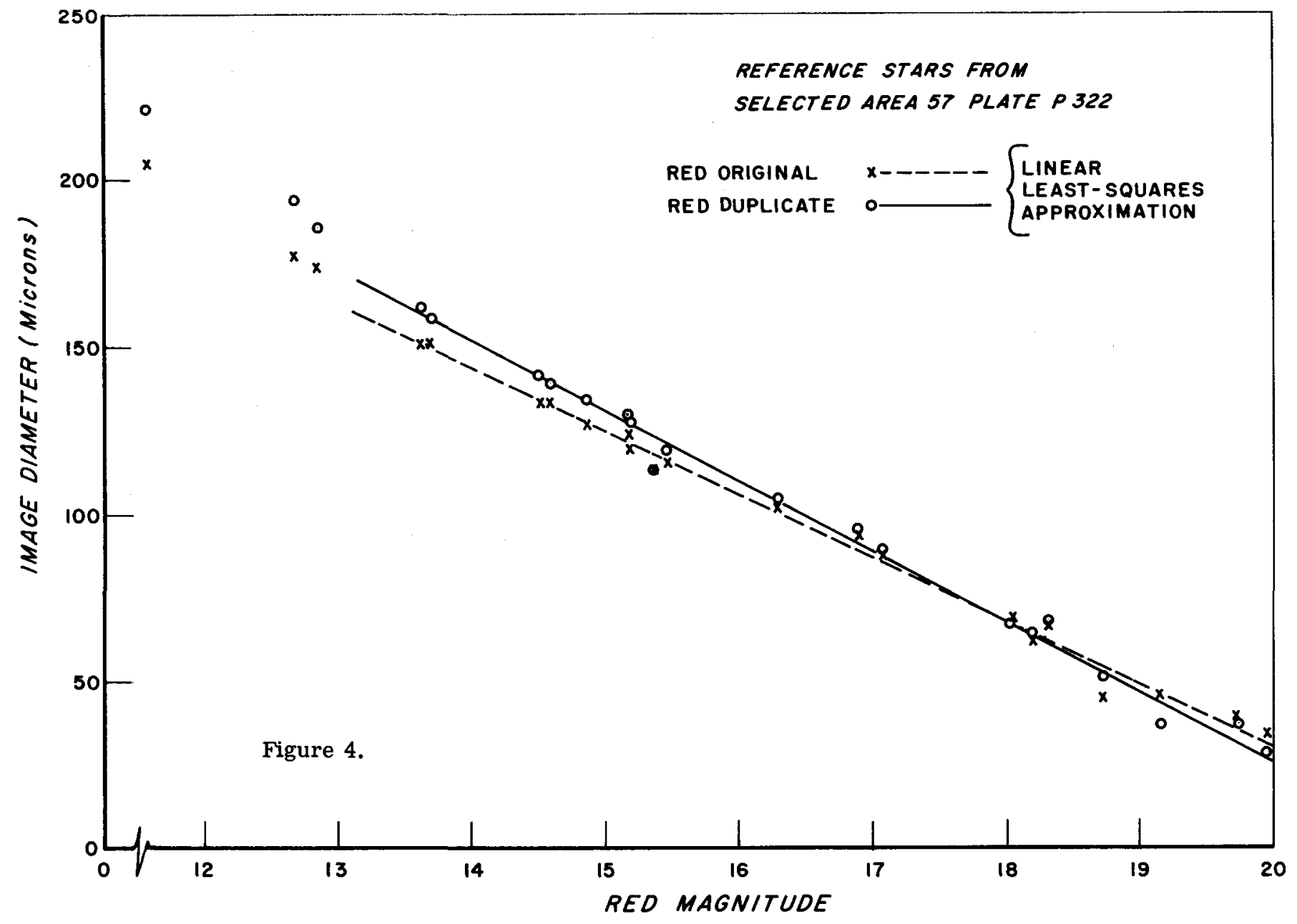


to infer red magnitudes for these stars ${ }^{(1)}$. The reduced scan data for the region was then searched to obtain the detected diameter of each reference star. Here, several scan passes were made with the star field in a number of different positions relative to the scan; each reference image was seen many times and at a variety of locations along the scan path.

The composite image diameter versus red magnitude results are presented in Figure 4. Note that the scanner is capable of reaching all the way to the accepted plate limit $\left(m_{r e d}=20\right)$ although the detections at this limit are less reliable than those above the survey cutoff at $m_{\text {red }}=19$. For both the original and duplicate plates, a linear relation between diameter and magnitude appears to be a very good approximation for stars fainter than thirteenth magnitude; the slope of the curves is approximately 20 microns per magnitude in this range. The standard deviation from the straight lines is 0.18 magnitude. This variation is due at least in part to the simplefflor equation used to convert the photoelectric data to red magnitudes ${ }_{\text {; }}$ it also reflects the inherent variability in the photographic acquisition of the stellar data. Finally, it is seen that the curves in Figure 4 turn upward for stars brighter than $m_{r e d}=13$. This is the range where diffraction spikes begin to show up and these larger images are processed in a special manner which deletes data from the region of the spikes and concentrates on the circular cores. Even so, non-linear photographic effects cause these brighter images to grow very rapidly with decreasing stellar magnitude. In any event, the magnitude sensitivity of the APMS system appears to be more than adequate for the survey limits.

To assess the measurement consistency of the system, a field containing 155 image pairs was scanned several times and the results analyzed in detail. The scan passes were arranged so that each image was seen at ten or eleven different locations along the y scan path. Further, the relative phase between the center of a particular image and the discrete grid of parallel scan lines was different for each complete scan pass. The system measurement performance on this sample is summarized in Table I; all entries are one-sigma values in microns.

Table I - APMS Measurement Performance

Standard Deviation of:

Old Duplicate New Photographic Original

$\begin{array}{llll}\mathrm{x} \text { center } & 1.52 & & 1.77 \\ \mathrm{y} \text { center } & 0.82 & & 1.17 \\ \text { diameter } & 0.89 & & 1.56 \\ \Delta \mathrm{x} \text { proper motion } & & 1.83 & \\ \Delta \mathrm{y} \text { proper motion } & & 1.22 & \end{array}$


Several conclusions can be drawn from these results. First, it may be noted that the $\mathrm{y}$ measures are somewhat better than those for $\mathrm{x}$. This is attributed to the fact that the scanner geometry measures y quantities directly while $x$ values must be inferred through a more elaborate process in which a specific model of the image (circular symmetry) is introduced. In all cases, however, the measurement consistency corresponds to approximately 0.1 arc sec on the plate; this is probably somewhat better than the inherent accuracy of the plate material in the exposure and photographic development processes.

Second, it is seen that the duplicate plate results are slightly more stable than those for the original plate. This appears to be due to the sharper image definition which results from the two added photographic processes.

Third, the diameter repeatability is found to correspond to better than 0.05 magnitudes on the duplicate and 0.08 magnitude on the original. This is a small fraction of the total variation observed in the magnitude reference results based on the SA 57 sequence. Thus, it may be concluded that the scanner and data reduction systems are not adding significantly to the magnitude uncertainties already inherent in the plate material.

Finally, the last two entries in Table I show that the proper motion determinations are consistent to somewhat better than 0.01 arc sec/year in each axis. This, of course, is the most important accuracy measure of the system; it is more than a factor of 2.5 times better than the performance called out in the original system specification.

With detection and measurement performance satisfactorily verified for typical individual images, examination of the APMS system turned to the processing of complete plate pairs. For this purpose, a region which had already xeen hand-blinked and manually measured was chosen. In particular, plate $\$ 439$ at $14^{h},+18^{\circ}$ (1855) was selected. The published data for this plate $(7)$ tabulates the blue magnitudes for the motion stars. Thus, the survey magnitude limits given in terms of red magnitudes cannot be applied directly in comparing the hand-blinked results with those obtained with the APMS system. Also, the manual measurements of the hand-blinked motions are made with a few nearby comparison stars while the reference system for each APMS motion measurement is based on several tens of "fixed" stars in the immediate vicinity. Thus, the hand-blinked versus APMS motion values are expected to show some statistical spread. As a consequence, all published motions greater than $0.08 \mathrm{arc} \mathrm{sec/year}$ with magnitudes in the range $10<\mathrm{m}_{\text {blue }}<21$ were selected as the a priori hand-blinked comparison sample; these extended ranges shoul $\bar{d}$ insure coverage of the corresponding APMS survey limits. 
A detailed search of the APMS output for P 439 revealed that $87.5 \%$ of the a priori hand-blinked comparison sample had been found. Those motions not found initially were then re-examined visually. Many of the misses were due either to the fact that the motion was too small (compare the a priori hand-blinked limit of $0.08 \mathrm{arc} \mathrm{sec} /$ year with the APMS limit of $0.10 \mathrm{arc} \mathrm{sec} / \mathrm{year}$ ) or to the fact that the star was too bright on the red plates (compare the a priori hand-blinked limit of $m_{\text {blue }}=10$ with the APMS limit of $m_{r e d}=12$ ).

Most of the remaining missed motions were due to some form of image contamination. Here, it may be noted that Arcturus lies within the field of $P 439$; it produces an image whose core is several tens of millimeters across with extensive photographic "bloom" beyond this central region. Several small motions were imbedded in this nearly opaque bloom and were not detected by the scanner. Arcturus also produces a strong ghost image in the $48^{\prime \prime}$ Schmidt Telescope and a few motions were obscured in the darker part of this ghost; motions were detected by the system, however, in the clearer central region of the ghost image. Fortunately, Arcturus represents very nearly the worst case in terms of bright image contamination on the red plates; few other stars in the sky will cause such severe problems.

Another source of contamination is associated with the large number of scratches on the P 439 plate material. These plates, of course, were hand blinked and measured several years ago $(7)$. They have also served as test plates throughout the development of the APMS scanner. Thus, they have undergone far more handling than any other plates to be used in the survey; the rest of the material appears to be in much better condition in terms of the density of scratches.

If the missed small motions, bright stars, and contaminated images are deleted, APMS then finds over $98 \%$ of the same motions found in the corresponding P 439 hand-blinked sample. The average motion agreement over this set is a remarkable $0.00015 \mathrm{arcsec} /$ year; this is clearly coincedental. The standard deviation in the motion agreement has the more reasonable value of $0.027 \mathrm{arc} \mathrm{sec} / y e a r$. This value is only slightly larger than the estimated internal consistency of the hand-blinked motions and is thus consistent, in turn, with the APMS estimates listed at the end of Table I.

Within the survey magnitude range, the APMS system finds nearly all of the stellar motions uncovered with the hand blink method. In addition, however, the automated system discovers a large number of new motions on the same plate material. For motions greater than $0.1 \mathrm{arcsec} /$ year, nearly half $(48 \%)$ of the validated APMS motions stars on P 439 were previously unknown. Further, if the motion limit were to be extended down to $0.08 \mathrm{arc} \mathrm{sec} / \mathrm{year}$, new motions would account for $65 \%$ of the total. 
Almost all of these additional entries exhibit motions less than 0.2 arc sec/year. This is the range where the hand-blinked results are acknowledged to be very incomplete; the image displacements between plates are small and are easily overlooked, particularly near the edges of the visual blink field. Once the motions have been discovered and pointed out by the APMS printout, however, they are sufficiently large to be readily verified by direct visual inspection. Thus, it is concluded that the impersonal detection capabilities of the APMS system should possess significant advantages for improving survey completeness.

APMS data for the comparisons described above was obtained from the output of the "global" conversion and assessment program. As noted earlier, this program must establish the transformation from plate to celestial coordinates on the basis of SAO catalog stars which are identified among the scan data. The region covered by plate $\mathrm{P} 439$ contains a total of 158 SAO stars. Of these, the program was able to recognize 47 among the list of successfully reconstructed bright images. The identified stars were well distributed in both $\mathrm{x}$ and $\mathrm{y}$; they thus provided a good basis for the calculation of the transformation.

The list of bright images is also used in another way by the assessment portion of the program. Since the bright images often exhibit a significant bloom in the background around them, the incidence of spurious detections may be markedly increased in the immediate vicinity. Further, since the bright image will be present on both plates, these "flaws" will be correlated and may result in the production of several invalid apparent motions. Thus, one of the assessment checks rates the motion candidates for proximity to bright images. Note that this test requires a list of all of the bright images whether they were successfully reconstructed or not. For the moderately bright stars (fainter than approximatly eighth magnitude), scan data is available and at least a crude center can be estimated which is sufficient to judge proximity. As a consequence, all detected bright images are placed on the list and a flag is set to denote those which were successfully reconstructed. Finally, scan data from the brightest stars may be completely rejected by the hardware transit length limit in the scanner; this is set at approximately one millimeter. These stars, however, are almost certainly in the SAO catalog and their plate centers can be adequately estimated by approximate inversion of the plate-to-celestial coordinate transformation.

Up to this point, the results of the APMS assessment program have been compared only with the hand-blinked results. This establishes a relative increase in survey completeness. The statistics of the APMS motions can also be analyzed internally to obtain a measure of the survey completeness as a function of motion size. The results for P439 are shown in Figure 5. 


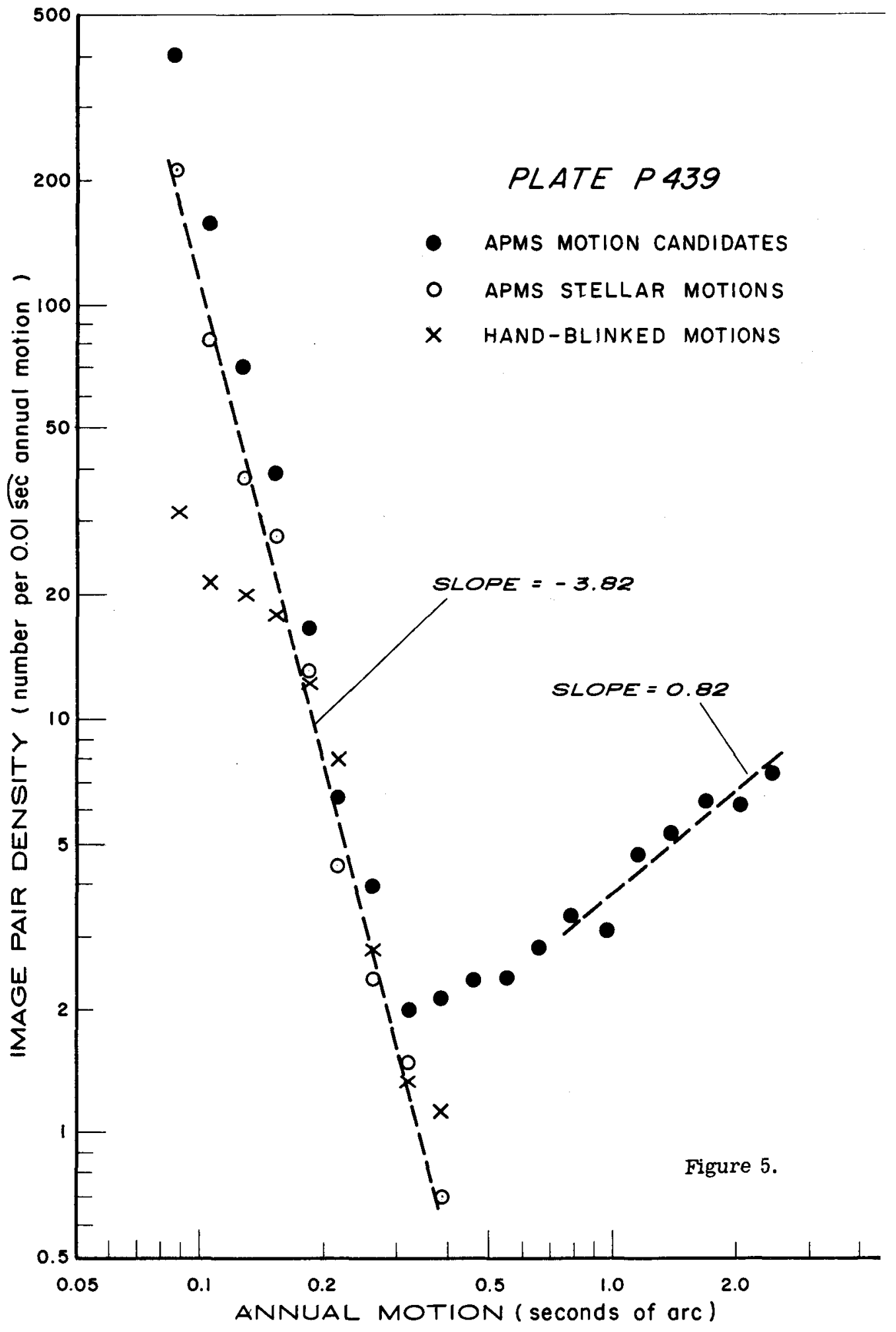


Here, the number density of APMS motion candidates, of net APMS stellar motions, and of hand-blinked motions is plotted as a function of the apparent motion.

The data for candidate motions is seen to possess a steep negative slope for small motions and a somewhat more gradual rise for larger displacements. Two broad classes of spurious apparent motions give rise to this dependence. At the small motion end, correct stellar pairs have been established but several "fixed" stars are attributed with apparent motion because of poor center determinations for one or both images. This may be due either to imperfection or contamination of the images themselves or to an occasional bad sample from the system. In either case, the image quality indices appear to yield a reliable indication of the error; such candidates are then rejected.

On the other hand, the large apparent motions are almost all the result of spurious matches. From a completely random distribution of unmatched images on the two plates, a linear increase in the pair density with separation would be expected. The slope of +0.82 in Figure 5 is in general agreement with this. Further, in the case of multiple matches, only the candidate with smaller displacement is retained if other factors are nearly equal. This plus the presence of some bright object correlation effects tends to increase the relative population at intermediate apparent motions; the observed slope of less than unity is thus quite reasonable. The diameter correlation obtained from the fixed star statistics is a powerful measure for assessment and rejection of erroneous matches which result in false large apparent motions. Note that this diameter correlation check is applicable to spurious stellar matches (where the quality indices of both images are good) as well as to star-flaw and flaw-flaw pairings. Of course quality-index and bright-objectproximity tests are also useful where at least one component of the pair is a recognizable flaw.

Application of these various assessments is effective in throwing out the vast majority of large candidate motions and only a very small set of potential large stellar motions will remain. All of these are assigned to the list of pairs to be visually verified by the astronomer since large apparent motions are both inherently suspect and of considerable individual importance. Usually no estimate as to the size of the motion is required; only the correctness of the pairing need be judged. The eye-brain combination is superbin this function and the decision can be made very quickly; the APMS system has carried out all of the tedious prior search and measurement operations.

The density of accepted APMS stellar motions is represented by the open circles in Figure 5. The data for the few legitimate motions greater 
than 0.4 arc sec/year has not been plotted because the sample size is too small for any reliable statistics. Both the APMS reduction and the handblinked search discovered the same pairings, though not identical displacements, for the large motions. It may be noted here that the APMS densities were plotted as a function of APMS motions while the hand-blinked densities are shown as a function of hand-blinked motion values. The rather marked discrepancy between the two stellar motion densities near $0.2 \mathrm{arc} \mathrm{sec} /$ year is a result of these different abscissa scales; the APMS motions were systematically smaller in this region.

The last and most interesting feature of the results in Figure 5 is represented by the density-versus-motion dependence of the APMS stellar motions. On the basis of the simple model that stellar distances are inversely proportional to the proper motion and that the spatial density of stars is uniform in the solar neighborhood, the density of proper motions would be expected to be proportional to the inverse fourth power of the motion itself. However, this does not take into account the fact that the stars also get relatively fainter with distance; the spatial density of detectable stars will thus fall off. This implies that the motion density should exhibit a slope somewhat less negative than minus four. Hence, the good straight line fit of the APMS stellar motion data and the remarkable slope of $\mathbf{- 3 . 8 2}$ for this line imply a high degree of completeness for the APMS results.

The evaluation described above represents only a small initial sample but the APMS performance appears quite impressive thus far. Two additional plate pairs - P 584 and P 240 - have been scanned and reduced through the main discovery and measurement program, APMSM. * Altogether on these first three plates, over 275,000 image pairs or 550,000 individual stellar images have been measured and checked for proper motion. Plates P 439 and $P 584$ are both sparse plates at fairly high galactic latitudes but P 240 is located at galactic latitude $+26^{\circ}$ and appears to be quite typical of a large fraction of the survey material. This latter plate contains about 160,000 image pairs and the APMSM reduction required less than one hour of central processor time on a CDC 6600 . Thus, images are being accurately reconstructed, matched between plates, and assessed for motion at the rate of approximately 100 images per second!

In summary, then, the conclusion that the APMS system meets its goals of accuracy (Table I), completeness (Figure 5), and efficiency is strongly supported by these initial performance results.

*As of April 21, 1970. 


\section{Acknowledgements}

The APMS system was developed for the National Aeronautics and Space Administration and the University of Minnesota by Control Data Corporation. Particular appreciation is extended to Dr. N.G. Roman and G. C. Augason of NASA for their interest and support throughout this development.

The successful design and implementation of APMS was the result of the dedicated efforts of a small group of individuals. Principal among these in the hardware area were J.S. Newcomb (optical system and APMS project engineer), Dr.G. E. Zenk (analog detection and control electronics), R. D. Willey (digital electronics), and W. Gysling (mechanical system). Much of the scanner calibration and initial operational evaluation were the responsibility of Dr. R. L. Cassola. Finally, the complex task of software implementation was carried out by B. D. Vannelli and H. R. Paetznick.

The entire program, of course, could not have been initiated or conducted without the vast astronomical knowledge, skill, and insight plus the tireless efforts of the principal investigator, Dr. W. J. Luyten.

\section{RE FERENCES:}

1. "The National Geographic Society - Palomar Observatory Sky Survey R.L. Minkowski and G.O. Abell, Appendix II, BASIC ASTRONOMICAL DATA, edited by K. A. Strand, pages 481-487, University of Chicago Press, 1963.

2. "Proper Motion Survey with the Forty-Eight Inch Schmidt Telescope, I. Organization and Purpose" Willem J. Luyten, The Observatory, University of Minnesota, 1963.

3. "The Luyten-Control Data Stellar Proper Motion Measuring Machine" J.S. Newcomb, Proceedings of IAU Colloquium No. 7 - Conference on Proper Motions, Minneapolis, Minnesota, 21-23 April 1970 (This is the preceeding paper in the present volume.)

4. SMITHSONIAN ASTROPHYSICAL OBSERVATORY STAR CATALOG, Smithsonian Institution, Washington D. C. , 1966 ( This publication includes a computer-compatible magnetic tape version of the catalog.)

5. "Photoelectric Magnitudes and Colors of Stars in Selected Areas 57, 61, and 68" J. Stebbins, A. E. Whitford, H. L. Johnson, Astrophys. J. 112 469-476 (1950). (Mt. Wilson magnitudes)

6. Mt. Palomar magnitudes, W. A. Baum, unpublished ( This data extends the SA 57 Magnitude Sequence down to the limits of the 200 inch Telescope.)

7. "Proper Mothion Survey with the Forty-Eight Inch Schmidt Telescope, III. Six Northern Regions", Willem J. Luyten, The Observatory, University of Minnesota, 1964. 


\section{DISCUSSION}

Wood: How do you deal with the possibility of magnitude equation, or prove that you do not have to deal with it because this may become important if the data are used statistically later.

La Bonte: This is a problem we have not yet fully resolved. We have found very few satisfactory reference star sequences against which we can calibrate the machine. In fact the only good one we know, that goes down to the plate limit, is SA 57. And we do not have any information on that particular area from more than one pair of plates. So one of the things that would be very useful to us would be to have the chance to process several plates with that same faint sequence on it.

Fricke: Would it not be surprising if a magnitude equation would occur at all for motions of this kind? I would expect that for displacements in the range from 15 to 500 microns actually nothing would happen. I think such effects are to be expected for much smaller motions, in particular if one takes into account the very fine quality of the Schmidt images.

Luyten: The Schmidt images are so completely symmetrical practically down to the plate limit that there is virtually no magnitude error. I have measured motions of 4th magnitude star against 21 st magnitude comparison stars and come up with the same answer as the G. C. So there cannot be much magnitude error.

Wesselink: What happens when you encounter a variable star?

La Bonte: The machine will probably reject it.

Hoffleit: In the general proper motion program, do you use exactly one pair of plates per region or more? We are very much interested actually in proper motions of variable stars and it would be a pity to lose them just because they happen to vary, and your diameter correlation does not work out well, so they get rejected. On the other hand, if you use more than one pair of plates, things would have a little better chance.

La Bonte: We do have one possible solution that would help that situation perhaps. That is, in addition ho the red plates which we examine we could, given enough money, redirect the effort to process three plates at a time, and get more information.

Hoffleit: On the other hand, your red and blue plates would probably be so closely the same eqoch that a variable star would not become evident. 
La Bonte: I meant that if we could guarantee, from the red and the blue plates, that we have a real stellar image then we would be much more likely to accept some other image as a mate to the first one whether the diameters agreed or not.

Luyten: In this connection I should like to point out that our machine has been designed to detect stars, and especially faint stars, with reasonably large proper motions. We do not intend to retain any motions smaller than, say, 0!'075 annually. This machine has not been designed to do all things for all men. Long-period variables, etc, fainter than the 12th magnitude are not likely to have motions larger than 0:'075 annually. Astronomers interested in those will have to design their own programs. With a program as extensive as this one - in which we expect that something like half a million proper motion stars will be found, we are not going to examine more than one pair of plates for each region - it might well mean another ten years even to take the second new plate.

Herget: I should like to make one more comment. Yesterday I had the advantage of all of you in that I had a private showing of the machine. I want to call your attention to what struck me in addition to everything else that will be said. This machine has a certain technical capability, but it has been directed solely down Luyten's alley. And many of us in astronomy will be interested in other uses of the technical capability than what you can find down Luyten's end.

Luyten: Our machine is an extremely versatile one - and can probably be adapted to do a great many other things in addition to detecting and measuring proper motions. After we have finishedour program, we will be glad to consider propositions to that effect. 